\title{
LIV-1 enhances the aggressive phenotype through the induction of epithelial to mesenchymal transition in human pancreatic carcinoma cells
}

\author{
JUN UNNO $^{1}$, KENNICHI SATOH ${ }^{1}$, MORIHISA HIROTA ${ }^{1}$, ATSUSHI KANNO ${ }^{1}$, SHIN HAMADA ${ }^{1}$, \\ HIROMICHI ITO $^{1}$, ATSUSHI MASAMUNE ${ }^{1}$, NOBUKAZU TSUKAMOTO ${ }^{2,3}$, FUYUHIKO MOTOI ${ }^{2}$, \\ SHINICHI EGAWA ${ }^{2}$, MICHIAKI UNNO ${ }^{2}$, AKIRA HORII ${ }^{3}$ and TOORU SHIMOSEGAWA ${ }^{1}$ \\ ${ }^{1}$ Division of Gastroenterology, Departments of ${ }^{2}$ Gastroenterological Surgery and ${ }^{3}$ Molecular Pathology, \\ Tohoku University Graduate School of Medicine, Sendai City, Miyagi, Japan
}

Received February 19, 2009; Accepted April 28, 2009

DOI: 10.3892/ijo_00000394

\begin{abstract}
LIV-1 is a downstream target of STAT3 and is essential for the nuclear localization of Snail, a master regulator of epithelial to mesenchymal transition (EMT). Little is known about the association of LIV-1 with pancreatic carcinoma development, therefore, expression of LIV-1 mRNA was analyzed by real-time reverse transcriptase polymerase chain reaction (RT-PCR) in 9 cultured cell lines ( 8 carcinoma and 1 normal duct cell lines) and 24 pancreatic tissues (12 carcinoma and 12 normal tissues). Localization of this gene product was investigated by immunohistochemistry in 72 pancreatic carcinoma and the relation between its expression and clinicopathological findings was examined. To assess the function of LIV-1 in pancreatic carcinoma cells, stable siRNA expressing Panc-1 cells were generated. Higher expression of LIV-1 mRNA was found in both pancreatic carcinoma cell lines and pancreatic carcinoma tissues compared to normal duct cell line and histologically normal tissues, respectively. Immunohistochemical analysis revealed that LIV-1 expression was frequently found in $76.4 \%$ of pancreatic carcinoma tissues and its expression level was associated with tumor size and lymphatic infiltration. Down-regulated LIV-1 cells showed significant inhibition of anchorage-dependent or -independent proliferation and cell motility in vitro and reduction of tumor growth and metastasis in vivo. Furthermore, nuclear expression of Snail was decreased and E-cadherin expression was restored in LIV-1 siRNA expressing pancreatic carcinoma cells. These findings indicate that LIV-1 may be involved in acquisition of the aggressive phenotype of human pancreatic carcinoma cells through the induction of epithelial to mesenchymal transition.
\end{abstract}

Correspondence to: Dr Kennichi Satoh, Tohoku University Graduate School of Medicine, 1-1, Seiryo-machi, Aobaku, Sendai City, Miyagi 980-8574, Japan

E-mail: ksatoh@mail.tains.tohoku.ac.jp

Key words: LIV-1, pancreatic cancer, epithelial to mesenchymal transition

\section{Introduction}

LIV-1 is a member of new subfamily of zinc transporters, termed LZT (LIV-1 subfamily of ZIP zinc transporters) and is suggested to be located to the plasma membrane. It has been shown to act as a zinc transporter, leading to an increase in intracellular zinc levels (1). Zinc plays various roles in cell metabolism and is involved in cellular processes such as cell growth, differentiation and gene transcription $(2,3)$, suggesting that its altered distribution might promote tumorigenesis. LIV-1 was originally identified as an estrogen-induced gene in the breast cancer cell line ZR-75-1 (4) and its expression was also shown to be correlated with lymph node involvement of the breast carcinoma, indicating a role for LIV-1 in metastasis (5).

Epithelial to mesenchymal transition (EMT) is a general phenomenon that is an essential event in embryonic development, tissue remodelling and wound repair. During EMT, a loss of intracellular adhesion is observed along with extensive remodelling of the cytoskeleton and expression of components specific to a mesenchymal phenotype. Epithelial cells undergoing the transition to a mesenchymal phenotype also display an increased propensity for migration (6-8). Therefore, this transition is considered to be an important event during malignant tumor progression and metastasis $(9,10)$. Previous study reveals that LIV-1 is involved in EMT of gastrula organizer cells in zebrafish by regulating the nuclear translocation of Snail, which has been shown to be master regulator of EMT through down-regulation of E-cadherin (11). In addition, LIV-1 is expressed in cervical cancer cells and inhibition of its expression by siRNA transfection resulted in the reduction of cell migration and invasion along with down-regulation of p44/42 MAPkinase posphorylation or Snail and Slug expression, suggesting that LIV-1 facilitates carcinoma cell invasion and metastasis (12).

Pancreatic cancer is one of the most malignant gastrointestinal tumors. Once pancreatic cancer is clinically evident, it progresses rapidly to develop metastatic lesions, frequently by the time of diagnosis. Although the pathogenic mechanisms that regulate the aggressive behavior of this cancer still remain to be clarified, EMT is possible mechanism to accelerate 
invasion or metastasis of pancreatic carcinoma cells. Little is known about LIV-1 expression or its association with EMT in pancreatic carcinoma. Therefore, we tested whether the presence of LIV-1 would correlate with the aggressive behavior of pancreatic cancer cells.

\section{Materials and methods}

Cell culture, RNA extraction and reverse transcriptionpolymerase chain reaction (RT-PCR) for cell lines. The following 8 pancreatic cancer cell lines were employed in this study: AsPC-1, BxPC3, Panc-1 and MIAPaca2 (American Type Culture Collection, Manassas, VA); KLM-1 (13), PK-45P (14), PK-9 (15), NOR-P1 (16) (Cell Resource Center for Biomedical Research, Tohoku University). These cells were grown in modified Eagle's medium (MEM, Invitrogen, Grand Island, NY) containing 10\% fetal bovine serum (Miles, Kankakee, IL), or in RPMI-1640 containing 10\% fetal bovine serum. Normal human pancreatic duct epithelium cells (HPDE) were kindly provided by Dr Ming-Sound Tsao, Ontario Cancer Institute and were cultured, as previously described (17). Cells were maintained at $37^{\circ} \mathrm{C}$ in $5 \% \mathrm{CO}_{2}$ in a humidified environment.

For cell RNA, total RNA was prepared using the RNeasy kit (Qiagen, Hilden, Germany) with DNase 1 treatment to eliminate DNA contamination according to the protocol provided by the manufacturer. First-strand cDNA was generated from $1 \mu \mathrm{g}$ total RNA using RETROscript (Ambion, Austin, TX) in a total volume of $20 \mu 1$ according to the manufacturer's protocol. To quantify the gene expression level, we also exploited quantitative real-time RT-PCR using LightCycler and LightCycler - FastStart DNA Master SYBR-Green I (Roche Diagnostics, Basel, Switzerland). All reactions were performed according to the manufacturer's protocol. The annealing temperature for these primer sets was $54^{\circ} \mathrm{C}$. The specificity of each PCR reaction was confirmed by melting curve analyses. The level of target gene expression in each sample was normalized to the respective GAPDH expression level. Each experiment was repeated at least three times and representative data are shown. The primer pairs used were LIV-1, forward 5'-ATGCCAGTGGTAGAAGGA ATG-3' and reverse 5'-CTTTTCGGGGCTCACTCACAG-3', GAPDH, forward 5'-GGCGTCTTCACCACCATGGAG-3' and reverse 5'-AAGTTGTCATGGATGACCTTGGC-3' and E-cadherin, forward 5'-GGTGGGTGACTACAAAATCAA TCT-3' and reverse 5'-TTCTCCGCCTCCTTCTTCATC ATA-3'.

Tissue samples. The study was approved by the Ethics Committee of Tohoku University Graduate School of Medicine, informed consent was obtained from each patients. Pancreatic cancer tissues were obtained from patients who underwent surgical operations for the tumors at the Tohoku University Hospital. The tissues collected at the time of surgery were immediately snap-frozen in liquid nitrogen and stored at $-80^{\circ} \mathrm{C}$ until used for RT-PCR analysis or fixed in $10 \%$ paraformaldehyde overnight and embedded in paraffin wax for tissue microarray (TMA) analysis. Total RNAs were extracted from frozen tissue samples (12 carcinoma and 12 histologically normal pancreas) using RNeasy kit (Qiagen) with DNase 1 treatment to eliminate DNA contamination according to the manufacturer's protocol. Quantitative real-time RT-PCR was performed, as described above. The level of target gene expression in each sample was normalized to the respective GAPDH expression level. Then, the LIV-1 expression level in each sample was normalized by the Panc-1 cell expression level.

Tissue microarrays. TMA blocks were constructed by punching out core sylinders of $3 \mathrm{~mm}$ from paraffin blocks after examination of slides. Each TMA contained up to 24 spots. Samples from one patient were mounted next to each other to confirm that the normal and tumor tissues of each patient were examined under the same condition. For analysis 12 TMAs were employed including 72 pancreatic carcinoma samples.

Immunohistochemistry. Paraffin sections were deparaffinized and antigens were retrieved by boiling the sections in Target Retrieval Solution (Dako, Carpenteria, CA) in a microwave oven. Then the sections were incubated in methanol with $0.3 \%$ hydrogen peroxide for $30 \mathrm{~min}$ in order to block the endogenous peroxidase activity. After treatment with $10 \%$ normal goat serum for $30 \mathrm{~min}$, the sections were incubated with the rabbit polyclonal to SLC39A6 (LIV-1) antibody (Abcam K.K., Tokyo, Japan) overnight at $4^{\circ} \mathrm{C}$. Thereafter, the histofine kit (Nichirei, Tokyo, Japan) was used and visualization of the

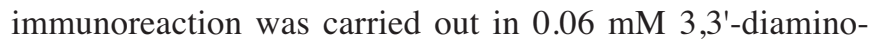
benzidine tetrahydrochloride (Dojin, Kumamoto, Japan) containing $2 \mathrm{mM}$ hydrogen peroxide in phosphate-buffered saline (PBS) for 3-4 min at room temperature. For the negative control, the immunostaining processes were performed by replacing the primary antibody with PBS. Fluorescent immunohistochemistry was also performed in representative pancreatic carcinoma tissues using the same antibody and detection was done as described below. The negative control sections showed no specific immunoreactivity.

The degree of immunostaining was evaluated as follows: negative, no positive cells were found; weak, $<10 \%$ of positive cells were observed; moderate, $10-50 \%$ of positive cells were observed; intense, $>50 \%$ immunoreactive cells were observed in the tissue sections. The evaluation of immunostaining was done independently by two observers (K.S. and J.U.) who had not been informed of the histological diagnosis.

RNA interference. The small interfering RNA for LIV-1 (LIV-1 siRNA) expressing vector was generated by cloning the following anneale BamHI and HindIII digested oligonucleotids into pBAsi-U6 Neo DNA vector (Takara Bio Inc., Ohtu, Japan): 5'-GATCCGTCACCACCATAGTCATA GTTCAAGAGACTATGACTATGGTGGTGACTTTTTTA-3' and 5'-AGCTTAAAAAAGTCACCACCATAGTCATAG TCTCTTGAACTATGACTATGGTGGTGACG-3' . This generates siRNA directed against the sequence GUCACCA CCAUAGUCAUAG, corresponding to nucleotide human LIV-1 at 1270 to 1288 (NCBI access number: NM_012319) under the control of the human U6 promoter. The LIV-1si expression vector or empty vector (EV) was transfected into Panc-1 cells using FuGENE 6 (Roche), as recommended by the supplier. After G418 selection, clones were subjected to RT-PCR to confirm LIV-1 expression. 
Western blot analysis. For whole cell protein extraction, cells were lysed by the addition of lysis buffer ( $50 \mathrm{mM}$ Tris- $\mathrm{HCl}$, $\mathrm{pH} 7.4,1 \%$ NP-40, $0.5 \%$ sodium deoxycholate). For nuclear protein, nuclear extraction was prepared using the NE-PER nuclear and cytoplasmic extraction reagents (Pierce Biotechnology Inc., Rockford, IL) according to the protocol provided by the manufacturer. After addition of $5 \mathrm{X}$ sample buffer (1 M Tris- $\mathrm{HCl}, \mathrm{pH}$ 6.8, SDS, glycerol and bromophenolblue) the aliquots were boiled for $5 \mathrm{~min}$ and subjected to $12.5 \%$ SDS-PAGE. After blocking for $1 \mathrm{~h}$ at room temperature in a buffer containing $10 \mathrm{mmol}$ Tris- $\mathrm{HCl}(\mathrm{pH} \mathrm{7.5),100 \textrm {mmol }}$ $\mathrm{NaCl}, 0.1 \%$ Tween-20 and 5\% dry milk, nitrocellulose membranes (Bio-Rad Laboratories, Hercules, CA) were incubated for whole cell protein extraction, with monoclonal mouse E-cadherin antibody (BD Biosciences), rabbit polyclonal LIV-1 antibody (Abcam), rabbit or polyclonal GAPDH antibody (Trevigen, Gaithersburg, MD), for nuclear protein extraction, either with polyclonal rabbit Snail antibody (Abcam) or polyclonal rabbit Lamin-B1 antibody (Santa Cruz Biotechnology, Inc., Santa Cruz, CA), overnight at $4^{\circ} \mathrm{C}$. The membranes were then washed with a buffer containing 10 mmol Tris- $\mathrm{HCl}$, pH 7.5, $100 \mathrm{mmol} \mathrm{NaCl}, 0.1 \%$ Tween-20 and incubated with anti-rabbit or mouse-immunoglobulin $\mathrm{G}$ coupled to peroxidase (Amersham Biosciences, Buckinghamshire, UK) for $1 \mathrm{~h}$ at room temperature. Reactive bands were detected using ECL chemiluminescence reagent (Amersham Biosciences).

Fluorescent immunohistochemistry. EV or LIV-1si-transfected Panc- 1 cells were grown to subconfluence on BD Falcon ${ }^{\mathrm{TM}}$ culture slides (BD Biosciences, San Jose, CA) and fixed with cold methanol (Wako, Osaka, Japan). After blocked with $0.1 \%$ bovine serum albumin in PBS, cells were incubated with mouse monoclonal E-cadherin antibody (BD Biosciences) or rabbit polyclonal Snail antibody (Abcam) overnight at $4^{\circ} \mathrm{C}$ and then slides were incubated with fluorescein-conjugated goat anti-mouse IgG (Jackson ImmunoResearch Laboratories. Inc., West Grove, CA) or fluorescein-conjugated goat antirabbit IgG (Jackson ImmunoResearch Laboratories.). Cells were then incubated with propidium iodide (Wako) for nuclear staining and mounted with Vectashield (Vector Laboratory, Inc.). Cells were visualized for immunofluorescence with BZ-8000 microscope (Keyence Co., Ltd., Osaka, Japan).

Cell growth assays. For the cell growth assay, 5,000 LIV-1sitransfected cells or EV cells were seeded per well in 96-well plates (Corning Incorporated, Corning, NY) in normal cell growth media. The 5-bromo-2-deoxyuridine (BrdU) assay was performed after 24, 48 and $72 \mathrm{~h}$ incubation using a kit (Roche) according to the manufacturer's protocol. For each cell line the proliferation index was evaluated and the absorbance at 48 and $72 \mathrm{~h}$ were normalized to that at $24 \mathrm{~h}$.

Soft agar assay. Transfected Panc-1 cells $\left(5 \times 10^{3}\right)$ were suspended in $0.3 \%$ Bacto $^{\mathrm{TM}}$ agar (BD Falcon) supplemented with DMEM medium containing $10 \%$ fetal bovine serum and layered over $1 \mathrm{ml}$ of an $0.8 \%$ agar-medium base layer in 6-well plates. After 21 days, the cells were stained with nitroblue tetrazolium (Roche) and anchorage-independent growth was estimated by counting the number of colonies using a microscope in high power view.

Scrape motility assay. Pancreatic cancer cells were grown to confluence in 12-well culture dishes (BD Falcon) with normal growth media. The cell monolayer was mechanically scarred with a sterile pipette tip and the plates were incubated for an additional 1 to 2 days. Cells were visualized with an Olympus Model CK2 inverted microscope using a 10X objective. Images were captured in a time-lapse manner with an Olympus C2000 a digital camera.

Tumor growth in nude mice. Tumor formation in vivo was assayed in female athymic nude mice by subcutaneously injecting each of $5 \times 10^{6}$ cells suspended in $100 \mu 1$ of sterile PBS. Tumor volume was measured every week after the first incidence of tumor formation. Volume was determined by the equation $\mathrm{V}=\mathrm{L} \mathrm{x} \mathrm{W}^{2} \times 0.5$ where $\mathrm{V}$ is volume, $\mathrm{L}$ is length and $\mathrm{W}$ is width. The mice were sacrificed 14 weeks after injection and confirming the histology by H\&E staining.

Orthotopic implantation. To assess metastasis formation, LIV-1si and empty vector-transfected pancreatic cancer cells $\left(1 \times 10^{6}\right.$ cells $/ 50 \mu 1$ of PBS) were injected into the pancreatic tail of female athymic nude mice. The mice were sacrificed 12 weeks after injection and tumor progression was confirmed.

Statistical analysis. The computer software Excel-Toukei 2004 (Social Survey Research Information Co., Ltd., Japan) was used for all statistical analyses. The correlation between LIV-1 expression and clinicopathological findings were analyzed by $\chi^{2}$ test or Fisher's exact test when the expected values in any of the cells is $<5$. The correlation between orthotopic injected mice and metastasis or peritoneal dissemination were analyzed by Fisher's exact test. The difference between two groups was statistically analyzed by unpaired t-test or Mann-Whitney U test. P-value of $<0.05$ was regarded as statistically significant.

\section{Results}

LIV-1 expression in pancreatic cancer and the correlation with clinicopathological findings. As shown in Fig. 1A, six of eight (75\%) pancreatic cancer cell lines expressed higher levels of LIV-1 mRNA compared to HPDE cells. Consistently, transcription level for LIV-1 was significantly higher in pancreatic cancer compared to normal tissues $(3.76 \pm 0.26$ vs. $0.54 \pm 0.17$, respectively, Mann-Whitney U test, $\mathrm{P}<0.01$, Fig. 1B). We then investigated the localization of LIV-1 protein in 72 human pancreatic carcinoma tissues and examine the correlation between its expression and clinicopathological findings. LIV-1 expression was detected in cytoplasm of carcinoma cells and occasionally in acinar cells and stromal cells neighboring the carcinoma cells while no or weak staining was seen in normal duct cells (Fig. 1C-F). The expression of LIV-1 was found in 55 of $72(76.4 \%)$ pancreatic cancer tissues. Among 72 cases of pancreatic carcinoma tissues 21 cases (29.1\%) were classified as intensely staining for LIV-1, 17 cases (23.6\%) were moderately stained, 17 cases $(23.6 \%)$ were weakly stained and 17 cases $(23.6 \%)$ were negative for staining. Significant 
A

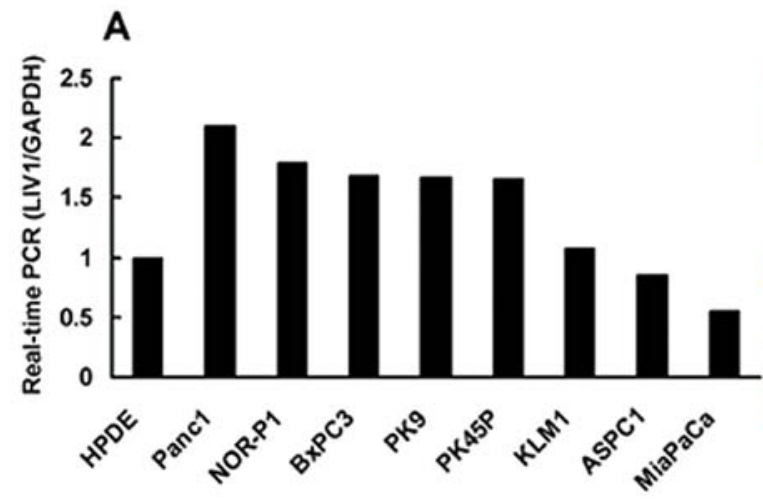

B

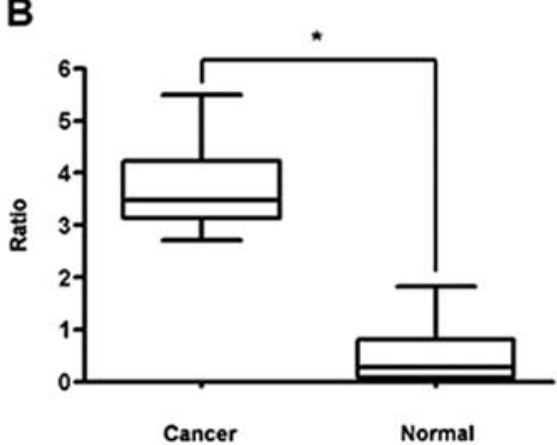

C

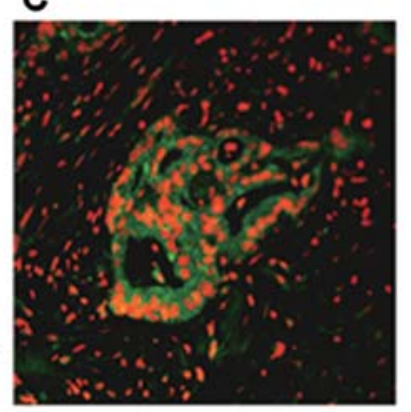

E

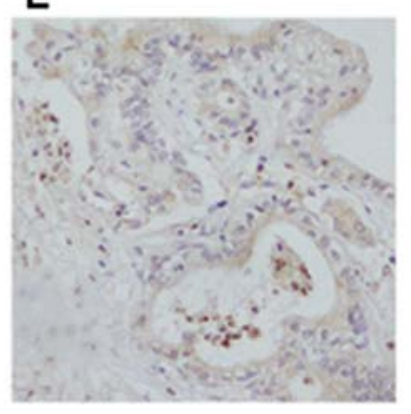

D

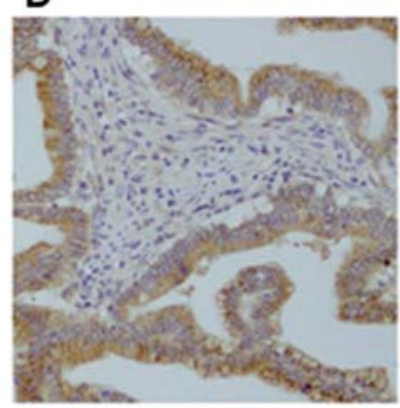

F

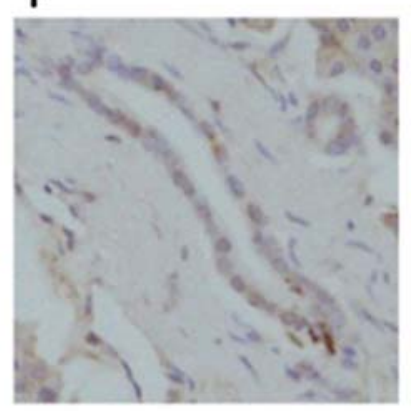

Figure 1. Expression of LIV-1 mRNA in pancreatic cancer cells. (A) LIV-1 mRNA expression levels in pancreatic cancer cell lines were investigated and compared to those of Human pancreatic duct epithelial (HPDE) cells by quantitative real-time RT-PCR. The expression levels of LIV-1 in 6 cancer cell lines (Panc-1, NOR-P1, BxPC-3, PK9, PK45-P and KLM-1) were more intense than that in HPDE cells. (B) The real-time RT-PCR analysis clearly showed stronger expression of LIV-1 in cancer tissues than in adjacent normal pancreas tissues. LIV-1 expression level in each samples was normalized by the corresponding GAPDH expression level. Values are expressed relative to 1.00 for expression in Panc-1 cells. ${ }^{*} \mathrm{P}<0.01$. (C-F) Expression of LIV-1 protein was investigated immunohistochemistry in human pancreatic carcinoma tissues. (C) Fluorescent immunohistochemistry showed the dominant LIV-1 expression in carcinoma cells compared to surrounding stromal cells. Nuclei were counterstained by PI (Propidium Iodide). The immunoreactivity for LIV-1 was positive in 55 of 72 pancreatic carcinoma samples as shown in (D) while 17 of 72 carcinoma tissues were judged as negative as demonstrated (E). (F) No or weak staining was seen in normal duct cells. Original magnifications, $\mathrm{x} 40$.

correlation was found between LIV-1 expression and tumor size $(\mathrm{P}<0.05)$ and lymphatic invasion $(\mathrm{P}=0.022)$ (Table $\mathrm{I})$. The results of immunohistochemistry for LIV-1 are summarized in Table I.

Generation of stable LIV-1-specific siRNA expressing cell lines and morphology of these cells. To confirm the role of LIV-1 in pancreatic cancer cells, we utilized Panc-1 cells that express high levels of endogenous LIV-1. These cells were stably transfected with a LIV-1-specific siRNA expression vector to down-regulate its expression. As shown in Fig. 2, we generated two clones of Panc-1 (P11 and P18) stably expressing LIV-1 siRNA. Significant reduction of LIV-1 expression in these transfectant (LIV-1si cells, P11 and P18) compared to control cells (Fig. 2A and B) was confirmed by quantitative real-time RT-PCR and Western blot analysis. A significant morphological difference was observed between LIV-1 siRNA (P11 and P18) and EV (P-EV)-transfected Panc-1 cells. As shown in Fig. 2B, P11 and P18 cells showed dense cell junctions relative to P-EV cells. The LIV-1 siRNA expressing cells, P11 and P18, demonstrated a more epithelial cell-like appearance compared to P-EV cells. This alteration of morphology resembled an epithelial phenotype rather than the usual phenotype of Panc-1.

Inhibition of LIV-1 expression by LIV-1 siRNA alters intracellular localization and expression level of E-cadherin and Snail in pancreatic cancer cells. To determine if downregulation of LIV-1 led to reversal of the EMT state, we examined immunofluorescent staining for E-cadherin as an epithelial marker and Snail as a regulator of EMT in LIV-1si cells or control cells. As shown in Fig. 3A, Panc-1 cells transfected with LIV-1 siRNA showed dominant membranebound staining of E-cadherin compared to P-EV (Fig. 3A). On the other hand, as shown in Fig. 3B, Snail was clearly localized in the nuclear in P-EV cells, while LIV-1si cells showed weakly diffuse distribution in the cytoplasm and nuclei (Fig. 3B). In addition, quantitative real-time RT-PCR and Western blotting showed increased E-cadherin expression in LIV-1si cells (Fig. 3C and D). Furthermore, nuclear expression of Snail was decreased in LIV-1si cells (Fig. 3E). These molecular changes implied that the reduced expression of LIV-1 shifted the Panc-1 cells from the mesenchymal to epithelial phenotype.

Down-regulation of LIV-1 suppressed cell proliferation and anchorage-independent cell growth of pancreatic cancer cells. In cell count assay, P11 and P18 cells showed $\sim 50 \%$ reduction of proliferation after $72 \mathrm{~h}$ of culture with normal medium and 60 to $70 \%$ reduction after $96 \mathrm{~h}$ compared to P-EV cells (72 h: $\mathrm{P}=0.043$ and $\mathrm{P}=0.0395$, respectively, $96 \mathrm{~h}: \mathrm{P}=0.0219$ and $\mathrm{P}=0.0249$, respectively) (Fig. 4A). In BrdU assay, P11 and P18 cells showed $\sim 40$ to $60 \%$ reduction of proliferation after $48 \mathrm{~h}$ of culture with normal medium and 65 to $75 \%$ reduction 
A

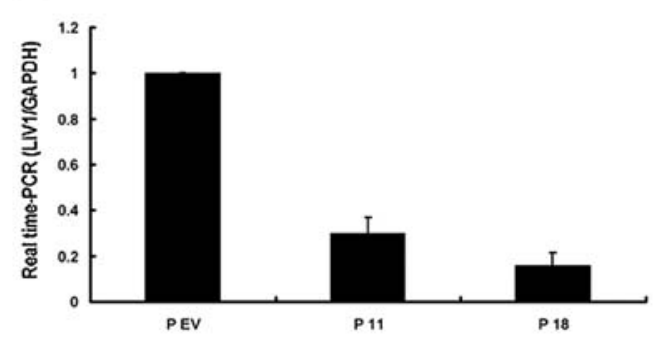

B

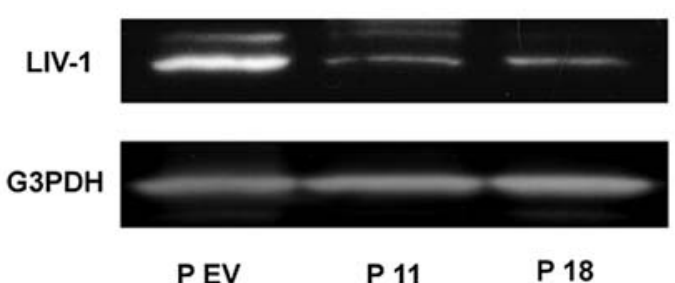

C
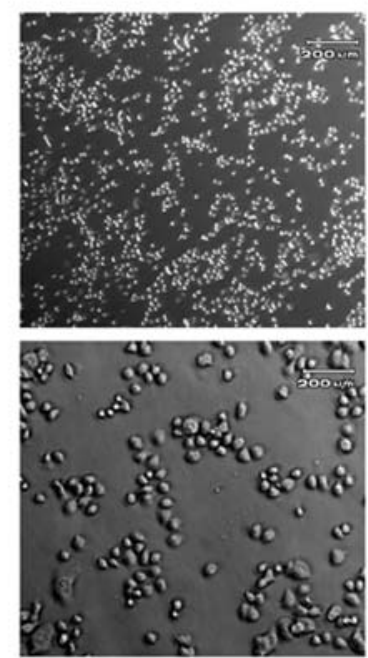

P EV
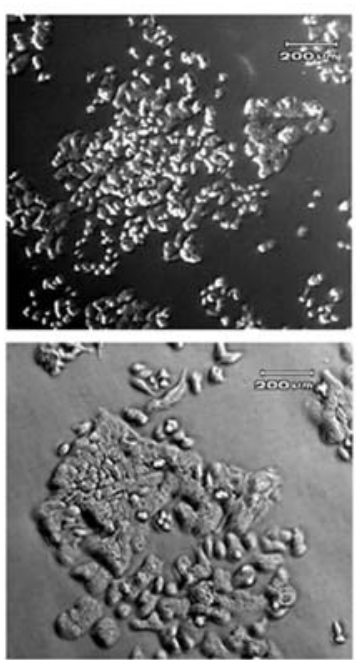

P 18

Figure 2. Generation of stable LIV-1 siRNA expressing cell lines and morphology of these cells. (A) Panc-1 cells were stably transfected with a LIV-1-specific siRNA expression vector to down-regulate its expression. LIV-1 expression level was investigated by triplicated real-time RT-PCR and normalized by the corresponding GAPDH expression level and then compared to vector-transfected control cells (P-EV). Significant reduction of LIV-1 expression was found in these transfectants (named P11 and P18) compared to control cells. (B) Reduced expression of LIV-1 protein in siRNA-transfected cells (P11 and P18) was confirmed by Western blot analysis. LIV-1 expression was decreased in P11 and P18 compared to control cells (P-EV). (C) LIV-1 down-regulated P18 cells showed dense cell junctions relative to P-EV cells, indicating that the LIV-1 siRNA expressing cell lines demonstrated a more epithelial cell-like appearance compared to P-EV cells.

A

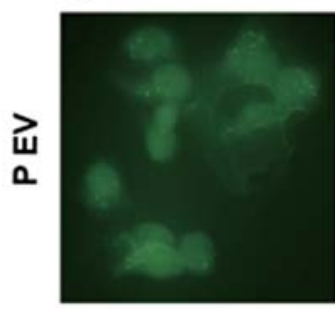

E-cadherin
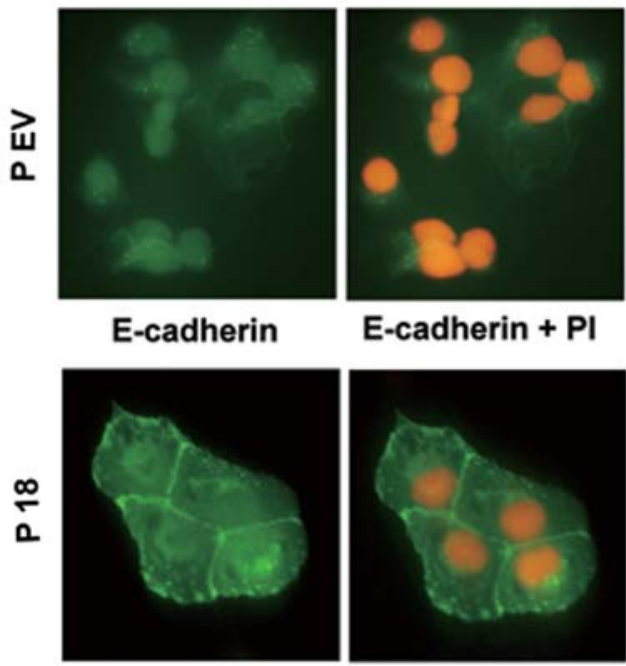

E-cadherin + PI

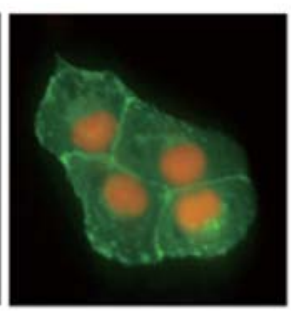

B

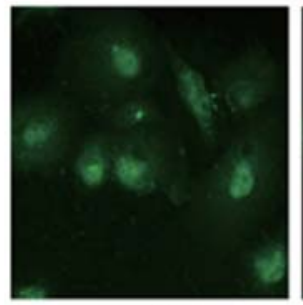

Snail

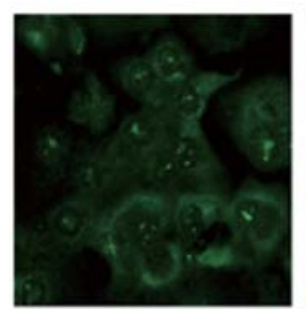

E

C

D
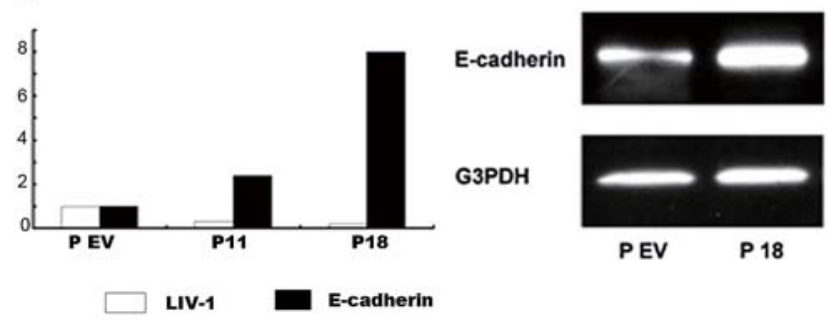

\section{Snail} nuclear

Snail

cytoplasm

Lamin B1

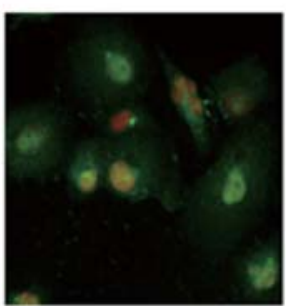

Snail + PI

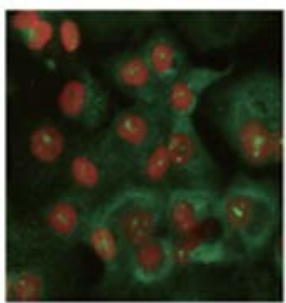

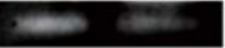
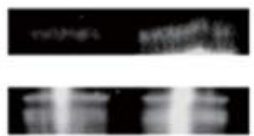

PEV

P 18

Figure 3. Molecular changes of siRNA-based LIV-1 knockdown in Panc-1 cells. (A) Fluorescent immunohistochemical staining for E-cadherin (A) and Snail (B) was performed in LIV-1 siRNA and EV-transfected Panc-1 cells. Panc-1 cells transfected with LIV-1 siRNA (P18) showed dominant membrane-bound staining of E-cadherin compared to P-EV cells (A). Snail was clearly localized in the nuclei in P-EV cells, while LIV-1si cells showed weakly diffuse distribution in the cytoplasm and nuclei (B). The real-time PCR analysis (C) and Western blot analysis (D) demonstrated that E-cadherin expression was restored in LIV-1sitransfected cells (P18). (E) Nuclear and cytoplasmic expression of Snail was confirmed by Western blot analysis. Nuclear expression of Snail was reduced and its cytoplasmic expression was increased in LIV-1 down-regulated pancreatic carcinoma cells (P18) compared to control cells. Lamin B1 was displayed as a loading control. 
Table I. Correlation between clinicopathological findings and LIV-1 expression.

\begin{tabular}{lll}
\hline LIV-1 staining & \\
\hline $\begin{array}{l}\text { Negative } \\
\text { to weak }\end{array}$ & $\begin{array}{l}\text { Moderate } \\
\text { to intense }\end{array}$ & P-value \\
\hline
\end{tabular}

\begin{tabular}{|c|c|c|c|}
\hline \multicolumn{4}{|l|}{ Age } \\
\hline$<60$ & 10 & 16 & $0.2629^{b}$ \\
\hline$\geq 60$ & 24 & 22 & \\
\hline \multicolumn{4}{|l|}{ Gender } \\
\hline Male & 25 & 24 & $0.3461^{\mathrm{b}}$ \\
\hline Female & 9 & 14 & \\
\hline \multicolumn{4}{|l|}{ Stage } \\
\hline $\mathrm{I}$ & 1 & 4 & $0.3567^{\mathrm{b}}$ \\
\hline II & 16 & 17 & \\
\hline III & 8 & 13 & \\
\hline IV & 8 & 5 & \\
\hline \multicolumn{4}{|c|}{$\mathrm{T}$ classification } \\
\hline $\mathrm{T} 1$ & 0 & 0 & $0.8228^{b}$ \\
\hline $\mathrm{T} 2$ & 3 & 5 & \\
\hline $\mathrm{T} 3$ & 17 & 19 & \\
\hline $\mathrm{T} 4$ & 14 & 14 & \\
\hline \multicolumn{4}{|c|}{ Lymph node metastasis } \\
\hline Negative & 9 & 13 & $0.4766^{\mathrm{b}}$ \\
\hline Positive & 25 & 25 & \\
\hline \multicolumn{4}{|c|}{ Histological classification } \\
\hline Well & 4 & 1 & $0.0701^{\mathrm{b}}$ \\
\hline Moderately & 23 & 34 & \\
\hline Poorly & 7 & 3 & \\
\hline \multicolumn{4}{|l|}{ Tumor size } \\
\hline$<50 \mathrm{~mm}$ & 30 & 26 & $0.0435^{\mathrm{b}}$ \\
\hline$\geq 50 \mathrm{~mm}$ & 4 & 12 & \\
\hline \multicolumn{4}{|c|}{ Lymphatic invasion } \\
\hline ly $(-)$ & 7 & 1 & $0.0227^{\mathrm{a}}$ \\
\hline ly $(+)$ & 27 & 37 & \\
\hline \multicolumn{4}{|c|}{ Vascular invasion } \\
\hline $\mathrm{v}(-)$ & 2 & 1 & $0.5992^{\mathrm{a}}$ \\
\hline $\mathrm{v}(+)$ & 32 & 37 & \\
\hline \multicolumn{4}{|c|}{ Perineural invasion } \\
\hline $\begin{array}{l}\text { ne (-) } \\
\text { ne }(+)\end{array}$ & $\begin{array}{r}2 \\
32\end{array}$ & $\begin{array}{r}1 \\
37\end{array}$ & $0.5992^{\mathrm{a}}$ \\
\hline
\end{tabular}

${ }^{\mathrm{a} A n a l y s e d}$ by Fisher's exact test. ${ }^{\mathrm{b}}$ Analysed by $\chi^{2}$ test.

after $72 \mathrm{~h}$ compared to $\mathrm{P}-\mathrm{EV}$ cells $(48 \mathrm{~h}: \mathrm{P}=0.0152$ and $\mathrm{P}=0.0035$, respectively, $72 \mathrm{~h}: \mathrm{P}<0.01$ and $\mathrm{P}=0.0008$, respectively) (Fig. 4B). These analyses clearly showed that down-regulation of LIV-1 significantly reduced the cell proliferation of pancreatic cancer cells. To elucidate the functions of LIV-1 in anchorage-independent growth of pancreatic cancer cells, we utilized the soft agar assay. P-EV cells expressing LIV-1 showed large size colonies and a large number of colonies on soft agar, while LIV-1si cells showed very few smaller colonies after 3 weeks of culture on soft agar (Fig. 4C and D). Three-fold and 6-fold more colonies
Table II. Summary of orthotopic implantation of LIV-1si cells (P18) or EV-transfected Panc-1 cells (P-EV) in nude mice.

$\mathrm{N} \quad$ Metastasis to liver or peritoneal dissemination

\begin{tabular}{llc}
\hline P-EV & 13 & 9 \\
P18 & 13 & $2^{\mathrm{a}}$ \\
\hline
\end{tabular}

${ }^{\mathrm{a}} \mathrm{P}<0.05$ (Fisher's exact test).

were seen in P-EV cells, compared to P11 and P18 cells $(\mathrm{P}=0.04$ and $\mathrm{P}<0.01$, respectively).

Down-regulation of LIV-1 inhibited the cell migration of pancreatic cancer cells. We next examined the cell migration ability of LIV-1si cells by a wound-healing scratch assay. As shown in Fig. 4E, P-EV cells covered the scratched area with migrating cells after $24 \mathrm{~h}$ incubation, but P18 cells did not (Fig. 4E), indicating that down-regulation of LIV-1 suppressed migration of pancreatic carcinoma cells.

Reduced expression of LIV-1 inhibits cell growth, metastasis and peritoneal dissemination in nude mice. To determine whether LIV-1si cells are also suppressed tumor growth in vivo, $5 \times 10^{6}$ of P-EV cells or LIV-1si cells (P18) were injected subcutaneously into the dorsal flank of nude mice. After 4 to 6 weeks from implantation, tumors began to appear in all P-EV injected and some of P18 injected nude mice. LIV-1si cells showed significantly slower growth and formed small tumors relative to those arising from EV cells (Fig. 5A and B). To assess whether reduced expression of LIV-1 also suppressed cell migration or metastasis formation in an orthotopic environment, $1 \times 10^{6}$ of P-EV cells or LIV-1si cells (P18) were injected into the pancreas of nude mice. Tumor formation was detected similarly in the pancreas of mice implanted with P-EV cells (8/13) and LIV-1si cells (9/13). Although there were no difference between the numbers of tumor formation, P-EV cells formed larger tumors than those of LIV-1si cells (data not shown). On the other hand, P-EV cells showed significantly more frequent metastases to the liver or peritoneal dissemination $(9 / 13)$ than LIV-1si cells $(2 / 13)(\mathrm{P}=0.0154)$ (Fig. 5C and Table II).

\section{Discussion}

This is the first study investigating the involvement of LIV-1 in human pancreatic carcinoma. We have clearly revealed that i) LIV-1 expression was more intense in most of pancreatic cancer cell lines compared to normal pancreatic duct cells (HPDE) and that the transcription level for LIV-1 was significantly higher in human pancreatic cancer compared to normal tissues, ii) $>75 \%$ of pancreatic carcinoma expressed LIV-1 protein and its expression was associated with lymphatic infiltration and tumor size, iii) down-regulation of LIV-1 in pancreatic carcinoma cells led to suppression of cell proliferation, anchorage-independent cell growth and cell migration ability in pancreatic cancer cells in vitro and iv) knockdown of LIV-1 inhibited tumorigenesis and metastasis or peritoneal dissemination in nude mice. 
A

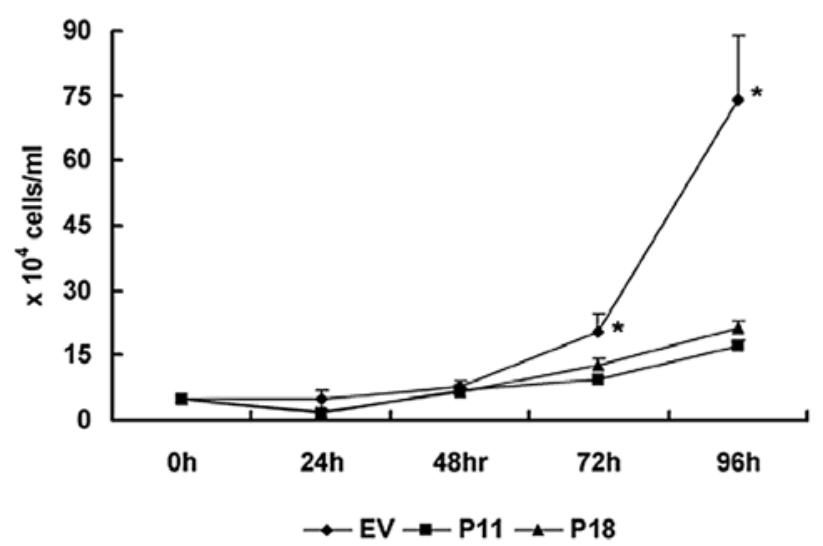

C P EV

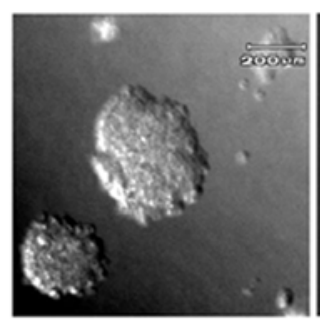

P 11
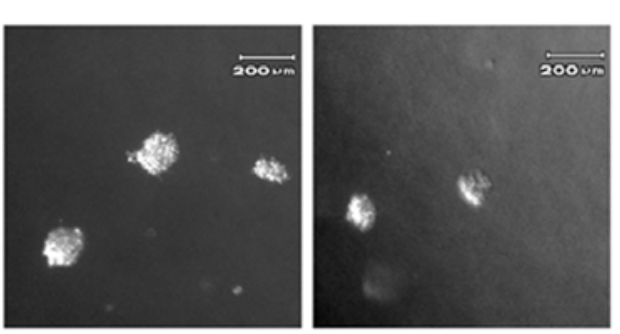

B

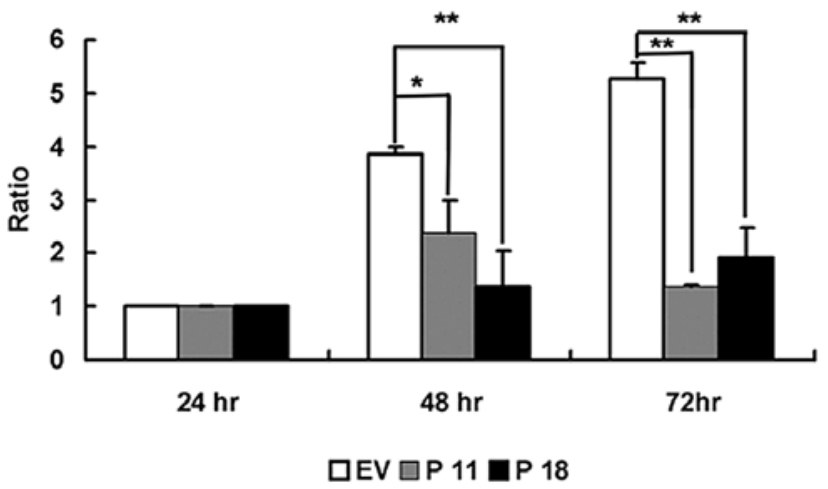

D

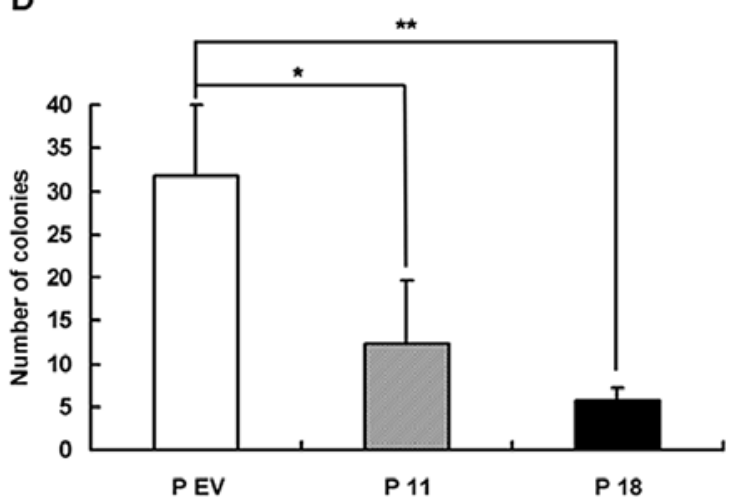

E

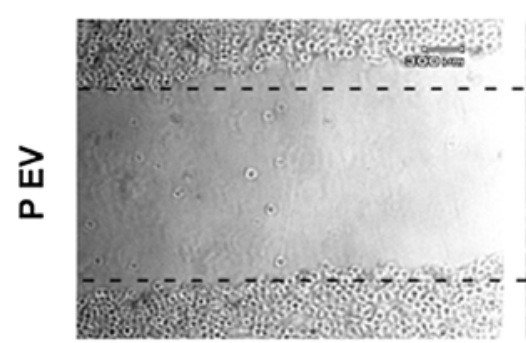

$0 \mathrm{hr}$

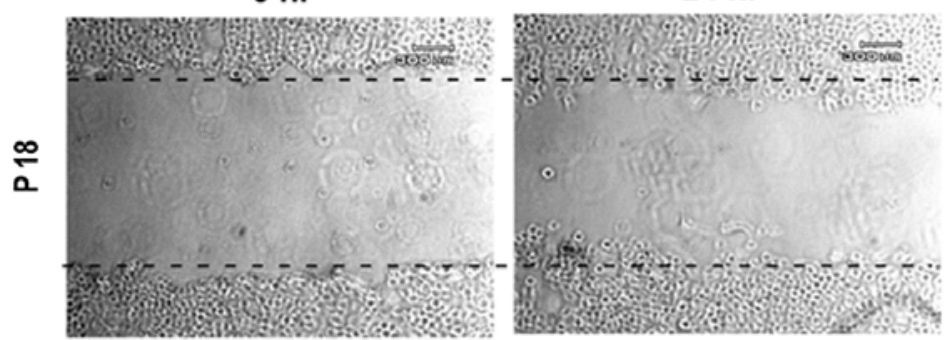

Figure 4. The effect of siRNA-based LIV-1 knockdown in Panc-1 cells. The cell growth was investigated by cell count (A) and BrdU assay (B). (A) LIV-1 knockdown cells (P11 and P18) showed $\sim 50 \%$ reduction of proliferation after $72 \mathrm{~h}$ of culture with normal medium and 60 to $70 \%$ reduction after $96 \mathrm{~h}$ compared to P-EV cells. "P<0.05 (B) LIV-1 down-regulated cells (P11 and P18) showed $\sim 40$ to $60 \%$ reduction of proliferation after $48 \mathrm{~h}$ of culture with normal medium and 65 to $75 \%$ reduction after $72 \mathrm{~h}$ compared to P-EV cells. ${ }^{*} \mathrm{P}<0.05 .{ }^{* *} \mathrm{P}<0.01$ (C and D). Five thousand of LIV-1si-transfected cells or P-EV cells were grown in soft agar for 3 weeks. Then the cells were stained with nitroblue tetrazolium and anchorage-independent growth was estimated by counting the number of colonies using a microscope with high power view. Three-fold and 6-fold more colonies were seen in P-EV cells, compared to LIV-1 down-regulated cells (P11 and $\mathrm{P} 18$ ), respectively. ${ }^{*} \mathrm{P}<0.05{ }^{* *} \mathrm{P}<0.01$. ${ }^{*}(\mathrm{E})$ Wound healing scratch assay clearly revealed that LIV-1si-transfected cells showed significantly slower migration and fewer migrated cells than the control P-EV cells.

The association of LIV-1 mRNA expression with lymph node involvement was also reported in breast cancer (5). Consistently, LIV-1 was expressed higher in carcinoma than in normal tissues and knockdown of LIV-1 by siRNA suppressed cell invasion in cervical carcinoma, suggesting the tumor promoting effect of this gene (12). In contrast, negative association of LIV-1 protein level with tumor size, grade and stage was reported in breast carcinoma tissues (18). In 


\section{A}

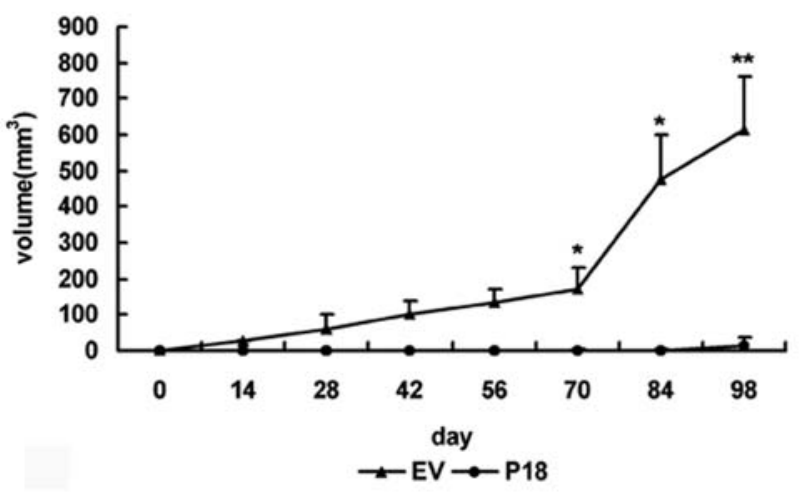

B

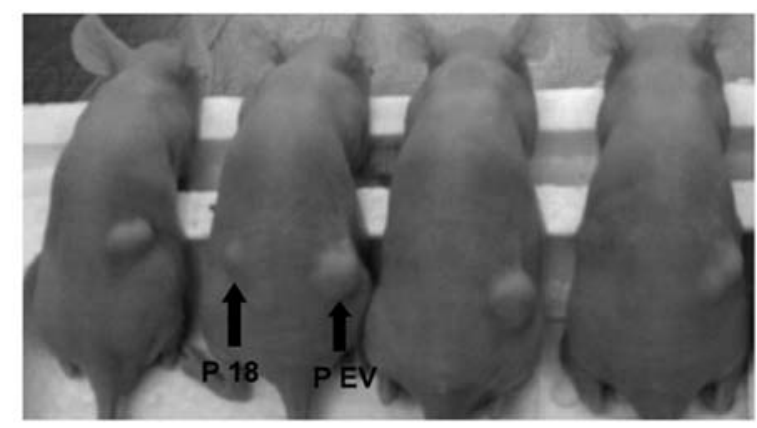

C

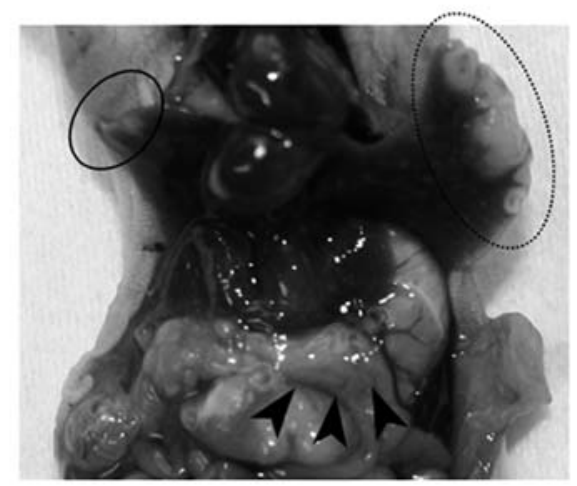

Figure 5. LIV-1 knockdown suppressed the tumor formation and the metastasis in nude mice. (A and B) Control (P-EV) cells $\left(5 \times 10^{6}\right)$ or LIV-1si cells (P18) were injected subcutaneously into the right and left side of each mouse, respectively. After 14 weeks, the mice were sacrificed. LIV-1si cells exhibited significantly slower growth as well as smaller tumor size in nude mice compared to P-EV cells. ${ }^{*} \mathrm{P}<0.05$. ${ }^{* *} \mathrm{P}<0.01$. (C) $\mathrm{P}-\mathrm{EV}$ cells injected into the pancreatic tail of nude mice showed more numbers of metastasis to the liver. Arrowheads show pancreatic tumor originated from the orthotopic graft, and metastatic liver tumor (left, solid line) and disseminated tumor lesion (right, dotted line) are encircled.

addition, expression levels of LIV-1 were associated with better outcome of these patients. The authors postulated that the discrepant result might be due to the tumor suppressive function of STAT3 occasionally observed in breast carcinoma since LIV-1 was downstream target of STAT3. In fact, nuclear expression of STAT3 has been shown to be associated with better 5-year survival of breast cancer patients (19). However, STAT3 was activated in pancreatic carcinoma and its inactivation inhibited cell growth, invasion and metastasis $(20,21)$, suggesting that STAT3 functions as tumor inducer but not tumor suppressor in pancreatic carcinoma. Taken together with our present results, LIV-1 is likely to contribute to tumor aggressiveness in pancreatic carcinoma.

Epithelial to mesenchymal transition (EMT) is a general phenomenon that is an essential event in embryonic development, tissue remodeling and wound repair. EMT is considered to be an important event during malignant tumor progression and metastasis $(9,10)$. EMT is characterized by the disassembly of cell-cell contacts, reorganization of the actin cytoskleleton and cell-cell separation by reduced E-cadherin expression. Zinc finger transcription factor Snail is shown to be a master regulator of EMT by transcriptionally repression of E-cadherin expression (22-24). Therefore, LIV-1 is considered to be involved in EMT since LIV-1 is shown to be required for activation of Snail (11). In the present study, we have demonstrated that LIV-1 siRNA expressing cell lines showed dense cell junctions, a more epithelial cell-like appearance, compared to control cells and that immunofluorescent staining as well as Western blotting showed dominant membranebound staining and increased expression of E-cadherin in LIV-1si cells. Snail was clearly localized in the nuclei of control cells, while LIV-1si cells showed weakly diffuse distribution in the cytoplasm and decreased nuclear expression of this protein. These molecular changes in LIV-1si cells indicate a reversal of the state of EMT, indicating that LIV-1 plays a pivotal role in EMT of pancreatic carcinoma cells. Thereby it is supposed that LIV-1 may lead pancreatic carcinoma cells to more malignant phenotype through the induction of EMT.

In addition to the function of LIV-1 as a regulator of EMT, we have demonstrated that LIV-1 expression was associated with tumor proliferation since reduction of LIV-1 expression in pancreatic carcinoma cells decreased in proliferation in vitro and in vivo. Although further studies would be required to validate the mechanisms, present finding of significantly higher expression of LIV-1 in large carcinoma tissues also supports the idea that LIV-1 promotes the growth of pancreatic carinoma cells.

To clarify the molecular mechanism for poor prognosis of pancreatic cancer patients, we have explored the factors involved in EMT and found that MSX2 (25) as well as periostin (26) was associated with EMT in pancreatic carcinoma cells and that MSX2 was indispensable for bone morphogenetic protein 4 (BMP4) induced EMT (27). Interestingly, down-regulation of MSX2 by siRNA or forced expression of low amount of periostin significantly reduced carcinoma metastasis to the liver in nude mice, respectively. Similarly, we have currently shown that suppression of LIV-1 resulted in the reversal of the state of EMT and the significant reduction of metastasis or dissemination of pancreatic carcinoma cells. Together these findings suggested that EMT might be one of key events of metastasis of pancreatic carcinoma and the blockade of this transition would be an effective therapeutic target of pancreatic carcinoma.

\section{Acknowledgements}

We thank Dr Ming-Sound Tsao for providing HPDE cells. This study was supported in part by Grant-in-Aid \#19590745 and \#20390202 from Ministry of Education, Science, Sports and Culture of Japan. 


\section{References}

1. Taylor KM, Morgan HE, Johnson A, Hadley LJ and Nicholson RI: Structure-function analysis of LIV-1, the breast cancer-associated protein that belongs to a new subfamily of zinc transporters. Biochem J 375: 51-59, 2003.

2. Fuchs O, Babusiak M, Vyoral D and Petrák J: Role of zinc in eukaryotic cells, zinc transporters and zinc-containing proteins. Sb Lek 104: 157-170, 2003.

3. Cousins RJ: A role of zinc in the regulation of gene expression. Proc Nutr Soc 57: 307-311, 1998.

4. Manning DL, Daly RJ, Lord PG, Kelly KF and Green CD: Effects of oestrogen on the expression of a $4.4 \mathrm{~kb}$ mRNA in the ZR-75-1 human breast cancer cell line. Mol Cell Endocrinol 59: 205-212, 1988.

5. Manning DL, Robertson JF, Ellis IO, Elston CW, McClelland RA, Gee JM, Jones RJ, Green CD, Cannon P and Blamey RW : Oestrogen-regulated genes in breast cancer: association of pLIV1 with lymph node involvement. Eur J Cancer 30: 675678, 1994.

6. Kiemer AK, Takeuchi K and Quinlan MP: Identification of genes involved in epithelial-mesenchymal transition and tumor progression. Oncogene 20: 6679-6688, 2001.

7. Kalluri R and Neilson EG: Epithelial-mesenchymal transition and its implications for fibrosis. J Clin Invest 112: 1776-1784, 2003.

8. Xue C, Plieth D, Venkov C, Xu C and Neilson EG: The gatekeeper effect of epithelial-mesenchymal transition regulates the frequency of breast cancer metastasis. Cancer Res 63: 3386-3394, 2003.

9. Thiery JP: Epithelial-mesenchymal transitions in tumour progression. Nat Rev Cancer 2: 442-454, 2002.

10. Petersen OW, Nielsen HL, Gudjonsson T, Villadsen R, Rank F, Niebuhr E, Bissell MJ and Rønnov-Jessen L: Epithelial to mesenchymal transition in human breast cancer can provide a nonmalignant stroma. Am J Pathol 162: 391-402, 2003

11. Yamashita S, Miyagi C, Fukada T, Kagara N, Che YS and Hirano T: Zinc transporter LIVI controls epithelial-mesenchymal transition in zebrafish gastrula organizer. Nature 429: 298-302, 2004

12. Zhao L, Chen W, Taylor KM, Cai B and Li X: LIV-1 suppression inhibits HeLa cell invasion by targeting ERK1/2-Snail/Slug pathway. Biochem Biophys Res Commun 363: 82-88, 2007.

13. Kimura Y, Kobari M, Yusa T, Sunamura M, Kimura M, Shimamura $\mathrm{H}$ and Matsuno S: Establishment of an experimental liver metastasis model by intraportal injection of a newly derived human pancreatic cancer cell line (KLM-1). Int J Pancreatol 20: 43-50, 1996.

14. Liu N, Furukawa T, Kobari M and Tsao MS: Comparative phenotypic studies of duct epithelial cell lines derived from normal human pancreas and pancreatic carcinoma. Am J Pathol 153: 263-269, 1998.

15. Kobari M, Hisano H, Matsuno S, Sato T, Kan M and Tachibana T: Establishment of six human pancreatic cancer cell lines and their sensitivities to anti-tumor drugs. Tohoku J Exp Med 150: 231-248, 1986

16. Sato N, Mizumoto K, Beppu K, Maehara N, Kusumoto M, Nabae T, Morisaki T, Katano M and Tanaka M: Establishment of a new human pancreatic cancer cell line, NOR-P1, with high angiogenic activity and metastatic potential. Cancer Lett 155 : $153-161,2000$
17. Furukawa T, Duguid WP, Rosenberg L, Viallet J, Galloway DA and Tsao MS: Long-term culture and immortalization of epithelial cells from normal adult human pancreatic ducts transfected by the E6E7 gene of human papilloma virus 16 . Am J Pathol 148: 1763-1770, 1996.

18. Kasper G, Weiser AA, Rump A, Sparbier K, Dahl E, Hartmann A, Wild P, Schwidetzky U, Castaños-Vélez E and Lehmann K: Expression levels of the putative zinc transporter LIV-1 are associated with a better outcome of breast cancer patients. Int J Cancer 117: 961-973, 2005.

19. Dolled-Filhart M, Camp RL, Kowalski DP, Smith BL and Rimm DL: Tissue microarray analysis of signal transducers and activators of transcription 3 (Stat3) and phospho-Stat3 (Tyr705) in node-negative breast cancer shows nuclear localization is associated with a better prognosis. Clin Cancer Res 9: 594-600, 2003.

20. Scholz A, Heinze S, Detjen KM, Peters M, Welzel M, Hauff P, Schirner M, Wiedenmann B and Rosewicz S: Activated signal transducer and activator of transcription 3 (STAT3) supports the malignant phenotype of human pancreatic cancer. Gastroenterology 125: 891-905, 2003.

21. Qiu Z, Huang C, Sun J, Qiu W, Zhang J, Li H, Jiang T, Huang K and Cao J: RNA interference-mediated signal transducers and activators of transcription 3 gene silencing inhibits invasion and metastasis of human pancreatic cancer cells. Cancer Sci 98: 1099-1106, 2007

22. Grau Y, Carteret $C$ and Simpson P: Mutations and chromosomal rearrangements affecting the expression of snail, a gene involved in embryonic patterning in Drosophila Melanogaster. Genetics 108: 347-360, 1984.

23. Batlle E, Sancho E, Francí C, Domínguez D, Monfar M, Baulida J and García De Herreros A: The transcription factor snail is a repressor of E-cadherin gene expression in epithelial tumour cells. Nat Cell Biol 2: 84-89, 2000.

24. Cano A, Pérez-Moreno MA, Rodrigo I, Locascio A, Blanco MJ, del Barrio MG, Portillo F and Nieto MA: The transcription factor snail controls epithelial-mesenchymal transitions by repressing E-cadherin expression. Nat Cell Biol 2: 76-83, 2000.

25. Satoh K, Hamada S, Kimura K, Kanno A, Hirota M, Umino J, Fujibuchi W, Masamune A, Tanaka M, Miura K, Egawa S, Motoi F, Unno M, Vonderhaar BK and Shimosegawa T: Upregulation of MSX2 enhances the malignant phenotype and is associated with twist 1 expression in human pancreatic cancer cells. Am J Pathol 172: 926-939, 2008.

26. Kanno A, Satoh K, Masamune A, Hirota M, Kimura K, Umino J, Hamada S, Satoh A, Egawa S, Motoi F, Unno M and Shimosegawa T: Periostin, secreted from stromal cells, has biphasic effect on cell migration and correlates with the epithelial to mesenchymal transition of human pancreatic cancer cells. Int J Cancer 122: 2707-2718, 2008.

27. Hamada S, Satoh K, Hirota M, Kimura K, Kanno A, Masamune A and Shimosegawa T: Bone morphogenetic protein 4 induces epithelial-mesenchymal transition through MSX2 induction on pancreatic cancer cell line. J Cell Physiol 213: 768-774, 2007. 